\title{
A Formal Framework for Bioprocesses in Living Cells
}

\author{
Andrzej Ehrenfeucht ${ }^{1}$ and Grzegorz Rozenberg ${ }^{1,2}$ \\ 1 Department of Computer Science, University of Colorado at Boulder \\ 430 UCB, Boulder, CO 80309, USA \\ 2 Leiden Institute of Advanced Computer Science, Leiden University \\ Niels Bohrweg 1, 2333 CA Leiden, The Netherlands
}

Natural Computing is an interdisciplinary field of research that investigates human-designed computing inspired by nature as well as computation taking place in nature. In other words, Natural Computing investigates models, computational techniques, and computational technologies inspired by nature as well as it investigates, in terms of information processing, phenomena/processes taking place in nature.

One of the fascinating research areas of Natural Computing is the computational nature of biochemical reactions taking place in living cells. It is hoped that this line of research may contribute to a computational understanding of the functioning of a living cell. An important step towards this goal is understanding interactions between biochemical reactions. These reactions and their interactions are regulated, and the main regulation mechanisms are facilitation/acceleration and inhibition/retardation. The interactions between individual reactions take place through their influence on each other, and this influence happens through the two mechanisms.

In our lecture we present a formal framework for the investigation of processes carried by biochemical reactions in living cells. We motivate this framework by explicitly stating a number of assumptions that hold for a great number of biochemical reactions, and we point out that these assumptions are very different from the ones underlying traditional models of computation. We discuss some basic properties of processes carried out by biochemical reactions, and demonstrate how to capture and analyze, in our formal framework, some biochemistry-related notions.

Besides providing a formal framework for reasoning about processes instigated by biochemical reactions, the models discussed in the lecture are novel and attractive from the computer science point of view.

The lecture is of a tutorial style and self-contained. In particular no knowledge of biochemistry is required. 